\title{
Role of Defects on Electronic Properties in Various Mono Layer Transition Metal Dichalcogenides
}

\author{
Ravinder Pawar ${ }^{1}$ \\ ${ }^{1}$ National Institute of Technology Warangal
}

February 16, 2021

\begin{abstract}
The chalcogen vacancy defects in various transition metal dichalcogenides (TMDCs) have been studied using density functional theory (DFT) calculation. Results reveal that (i) the dissociation energy value depends on both nature of chalcogen and transition metal, (ii) the work function depends marginally on the single or double vacancies, (iii) the defect transforms direct band gap to indirect band gap materials (i.e. the pristine materials show KV?KC transition whereas defective materials show $\Gamma \mathrm{V}$ ?KC) and (iii) the d-orbital of the transition metal plays a vital role in the formation of impurity band.
\end{abstract}

\section{Hosted file}

MS.pdf available at https://authorea.com/users/396129/articles/509282-role-of-defects-onelectronic-properties-in-various-mono-layer-transition-metal-dichalcogenides 